\title{
PRES: The Controlled Noninvasive Stimulation of the Carotid Baroreceptors in Humans
}

\author{
Harald Rau, Thomas Elbert, Bertram Geiger, and Werner Lutzenberger \\ Clinical and Physiological Psychology, Eberhard-Karls University. Tübingen, and University of Konstanz, Germany
}

\section{ABSTRACT}

To study physiological and psychological effects of baroreceptor activity, the cervical neck cuff technique has been frequently used to stimulate the carotid baroreceptors mechanically. Using this technique, no satisfying control conditions to date have been available.

Because the carotid stretch receptors are sensitive not only to the pressure level, but also to the rate of change, it is possible to manipulate the receptor firing through changes in carotid pulse amplitude. The device described here relies on the application of short changes in cuff pressure tied to different phases within the cardiac cycle (phase related external suction (PRES)). A brief external suction during systole has potent stimulatory effects on baroreceptors whereas the application of the very same pressure pulse during diastole inhibits the firing burst associated with the pulse wave. To allow an ongoing period of stimulation, a sequence of alternating negative/positive pressure pulses is applied. In the stimulation condition, the R-wave of the electrocardiogram triggers a negative pulse which is followed by a positive one during diastole. In the control condition this relationship is reversed.

Two experiments are reported confirming different baroreceptor effects of the two conditions. PRES allows for blind or double-blind experiments to investigate effects of baroreceptor activity on physiology and behavior.

DESCRIPTORS: Baroreceptors, Baroreceptor stimulation, Cervical neck suction, PRES.

The Laceys' hypotheses (Lacey \& Lacey, 1970) describe a feedback pathway from the cardiovascular system to the CNS by which primarily inhibitory effects on cortical structures are modulated. This negative feedback is transmitted by the baroreceptors that act on the brainstem vasomotor systems from which activity ascends to higher, including cortical, regions. These hypotheses led to further studies of the extrahomeostatic effects of baroreceptor activation.

The arterial baroreceptors are stretch receptors, located in the wall of the carotid sinus and the aortic arch. Increased blood pressure stretches the walls of the vessels and consequently stimulates the baroreceptors. Their afferent input to the cardiovascular brainstem center lowers blood pressure by reducing heart rate (negative chronotropic effect), the

This research was supported by the Deutsche Forschungsgemeinschaft (EL 101/3). We gratefully acknowledge the assistance in editing the paper of Lesslie Prewitt and Dr. Anthony Caravan, and the laboratory assistance of Renate Schweizer, Harald Schupp, and Ping Zhuang.

Address requests for reprints to: Harald Rau, Clinical and Physiological Psychology, Eberhard-Karls-University, Gartenstrasse 29, D 7400 Tübingen, Germany. contractility of the heart (negative inotropic effect), and vascular tone (Kirchheim, 1976).

To uncover possible behavioral effects of baroreceptor activity, several authors (e.g., Dworkin, Filewich, Miller, Craigmyle, \& Pickering, 1979; Randich \& Maixner, 1984; Larbig, Elbert, Rockstroh, Lutzenberger, \& Birbaumer, 1985; Schweizer, Roth, \& Elbert, 1991) manipulated blood pressure pharmacologically and observed behavioral and physiological changes as dependent variables (for a summary see Dworkin, 1988). To separate undesired direct behavioral effects of the drugs from desired direct baroreceptor effects, surgical denervation of the arterial baroreceptors in animals served as the control condition in the studies of Dworkin et al. (1979) and Randich and Maixner (1984). Differences between the behavioral responses to blood pressure increases among animals who underwent a sham operation (in which the baroreceptors were left intact) and those who underwent a surgical denervation (in which the baroreceptors were destroyed) were seen as evidence for direct effects of baroreceptor activity. Because this technique is possible only in animal research, these studies do not allow any conclusions regarding physiological conditions in humans. 
In humans, it is difficult to assess whether behavioral effects are mediated by changes in blood flow or by the direct effect of drugs on CNS structures. Vaitl and Gruppe (1990), using the tilting technique of the body, in which the head is tilted downward to enable the loading of the baroreceptors, observed increased theta activity in the EEG. Although these authors creatively arranged various control procedures, a totally convincing control still remained to be developed.

Another means of manipulating baroreceptor firing mechanically in humans relies on cervical neck suction, an approach that has been used recently for psychophysiological investigations (Toon, Bergel, \& Johnston, 1984; Elbert et al., 1988; Rau et al., 1988). In these studies, air pressure was manipulated in a cuff around the neck (see Figure 1a), as developed by Eckberg, Cavanaugh, Mark, and Abboud (1975). An air pump, connected to the cuff, controlled the extracervical pressure, i.e., the pres-

a

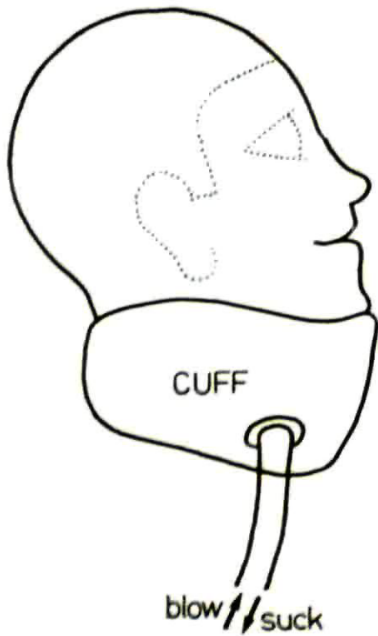

b

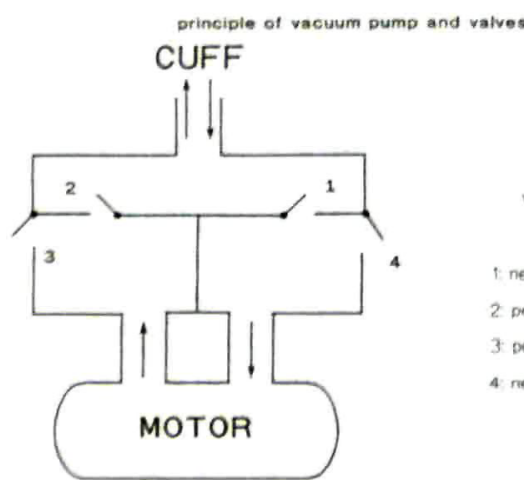

Figure 1. a. Sketch of the neck cuff used in the present experiment. b. Principle of the stimulation device: four switchable valves transmit the air flow through the motor to the air chamber in the neck cuff. sure on the tissue surrounding the carotid sinus. The baroreceptors are stretch receptors, which respond to extensions of the arterial wall. Any variance in pressure between the inside and the outside of the blood vessel alters the firing rate of the baroreceptors. The receptors cannot monitor whether such pressure changes occur inside the vessel (resulting from changes in blood pressure) or outside in the surrounding tissue. A negative extracervical pressure (negative cuff pressure) increases the transmural pressure difference and, hence, enhances baroreceptor activity, whereas a positive cuff pressure causes an inhibition. Mancia et al. (1977) reported that on the average $86 \pm 2 \%$ of positive and $64 \pm 3 \%$ of negative cuff pressures are transmitted to the tissues adjacent to the carotid sinus. This neck suction technique has been found to enhance baroreceptor activation reliably, as validated through heart rate decelerations in response to application of negative cuff pressure, systematic changes in digital pulse amplitude, and T-wave amplitude of the electrocardiogram (Toon et al., 1984; Elbert et al., 1988; Rau et al., 1988).

Previously, the application of the neck suction technique has had one fundamental limitation: There was a lack of a psychological control condition. In the studies of Toon et al. (1984), Elbert et al. (1988), and Rau et al. (1988), effects of negative cuff pressure were contrasted with effects of positive cuff pressure. Differences in negative and positive cuff pressures, however, seemed not to be confined to differential effects on the baroreceptors but also had differential effects on receptors in the skin, larynx, or pharynx etc., and were related to different sensations. Consequently, both the psychological and physiological processes may differ due to exteroceptive influences.

To overcome this critical disadvantage, we designed a neck suction procedure that allowed for both baroreceptor stimulation and a control condition that could not be discriminated solely on the basis of exteroceptive information alone. The basic idea for this technique was developed in cooperation with B. Dworkin (1988). The technique used was based on the finding that carotid stretch receptors are sensitive not only to static levels of pressure, but also to the rate of change of pressure. Consequently, the same suction pulse will have different effects when applied to different segments within the cardiac cycle (Eckberg, 1976; Dworkin, 1988). We will refer to the technique as PRES (cardiac phase related external suction): Short bursts of either negative or positive cuff pressure are applied during either systole or diastole. Application of a 200-ms duration negative pressure burst during systole adds to the pulse wave triggered dilation of the 
vessel and enhances both the speed and the degree of the dilation. As a result, baroreceptors will fire more vigorously. The application of the same 200$\mathrm{ms}$ duration negative cuff pressure burst during diastole will not overlap with systolic dilation: It will only delay the recession of the arterial stretch. Because baroreceptors respond not only to the static levels but also to differential aspects, systolic suction can be expected to enhance baroreceptor activation more effectively than diastolic suction. Figure 2 shows the theoretical relationship between the cardiac cycle, the pulse wave, and the consequence of cuff pressure bursts during systole and diastole.

In a number of pilot studies, it became clear that suction bursts alone are not sufficient to evoke great enough heart rate differentiations exceeding 1-2 bpm; therefore, the addition of a positive pressure burst is necessary. The excess pressure pulse should follow the suction: It should be applied during diastole in the case of systolic suction and during systole in case of diastolic suction. The first situation enhances the amplitude of the pulse wave triggered

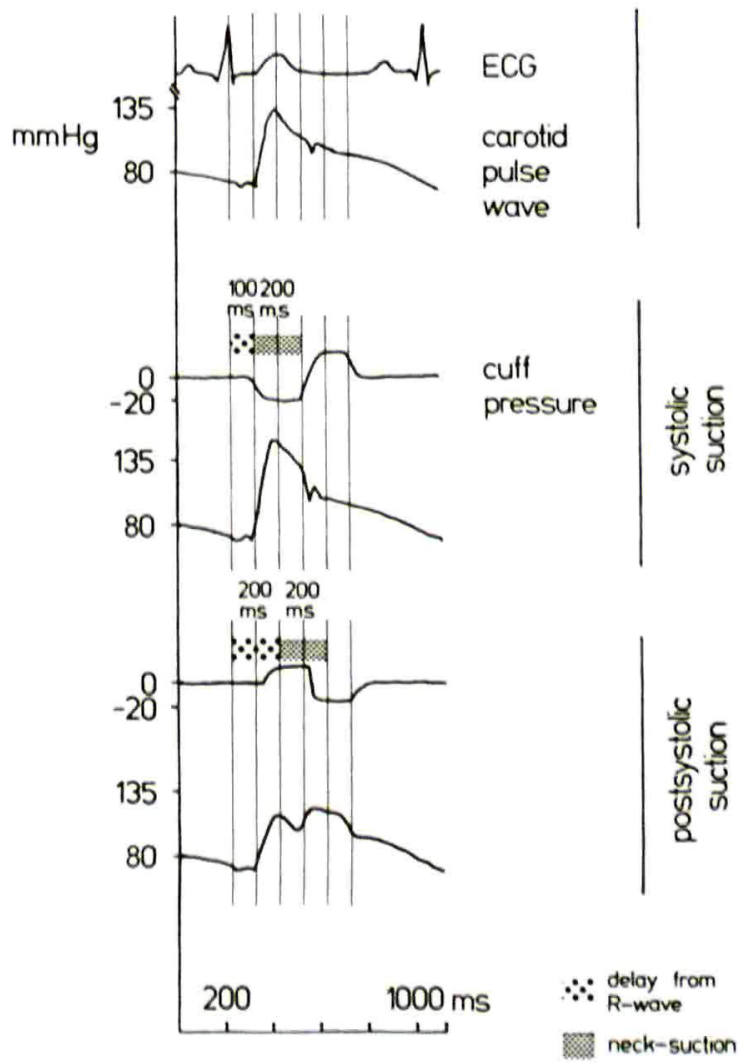

Figure 2. Theoretical relationship among electrocardiogram (ECG), carotid pulse wave, and cuff pressure (simulated data). Systolic negative cuff pressure enhances the carotid pulse wave, postsystolic negative cuff pressure has no influence on the transmural pressure changes caused by the pulse wave. dilation, the second flattens the effect of the internal pulse wave.

In the following, the technical details of PRES are described and its experimental effects on heart rate responses are examined.

\section{Technical Details}

The neck cuff is shown in Figure 1a. The cuff, which is made of lead, can be easily formed into shape so that it fits to the particular anatomical conditions. The metal is varnished to prevent any contact between the toxic material and the subject's skin. (See Eckberg et al., 1975, for an exact description of the neck cuff). Two tubes connect to the airtight chamber between the cuff and the neck. One tube serves to monitor the actual pressure inside the cuff. The other builds the connection to the pressure generator, which consists of a system of computer controlled valves and an air pump. A motor removed from an ordinary vacuum cleaner serves as an air pump. The speed of the motor and hence the pressure generated can be adjusted manually. The motor itself can be switched on/off through a relay, controlled via the digital output of a computer. The air pump changes the pressure in a system of pressure chambers, regulated through four computer controlled valves. Each of these valves has a surface area of $13 \mathrm{~cm}^{2}$ and is attached to both an electromagnet and a spring-clip. (The electromagnets open the valves in less than $100 \mathrm{~ms}$ and a release of the magnet closes them.)

Figure $1 \mathrm{~b}$ illustrates the principle of this machinery, Figure 3 the more detailed specifications. The motor runs continuously at a constant speed. In the neutral state Valves $\mathbf{3}$ and $\mathbf{4}$ are open, and Valves 1 and 2 are closed. In this position air is pumped through the system without affecting the atmospheric pressure within the cuff. To transduce a negative pressure to the cuff, Valves 3 and 1 are opened while Valves 2 and 4 are simultaneously closed. If, on the other hand, Valves 2 and 4 are opened and Valves 1 and 3 are closed, a positive pressure is transmitted to the chamber around the neck. With this technique the cuff pressure can be changed rapidly; $180 \mathrm{~ms}$ after the valves are switched, about $2 / 3$ of the final cuff pressure is achieved. The motor was adjusted so that negative pressure bursts reach about $-30 \mathrm{mmHg}$ and positive bursts about $+10 \mathrm{mmHg}$.

\footnotetext{
'Although a negative pressure presses the cuff onto the skin, and thus automatically tightens gaps between cuff and tissue, an excess pressure increases these gaps and thus enlarges air leakage. Therefore high positive pressures are technically more difficult to accomplish.
} 

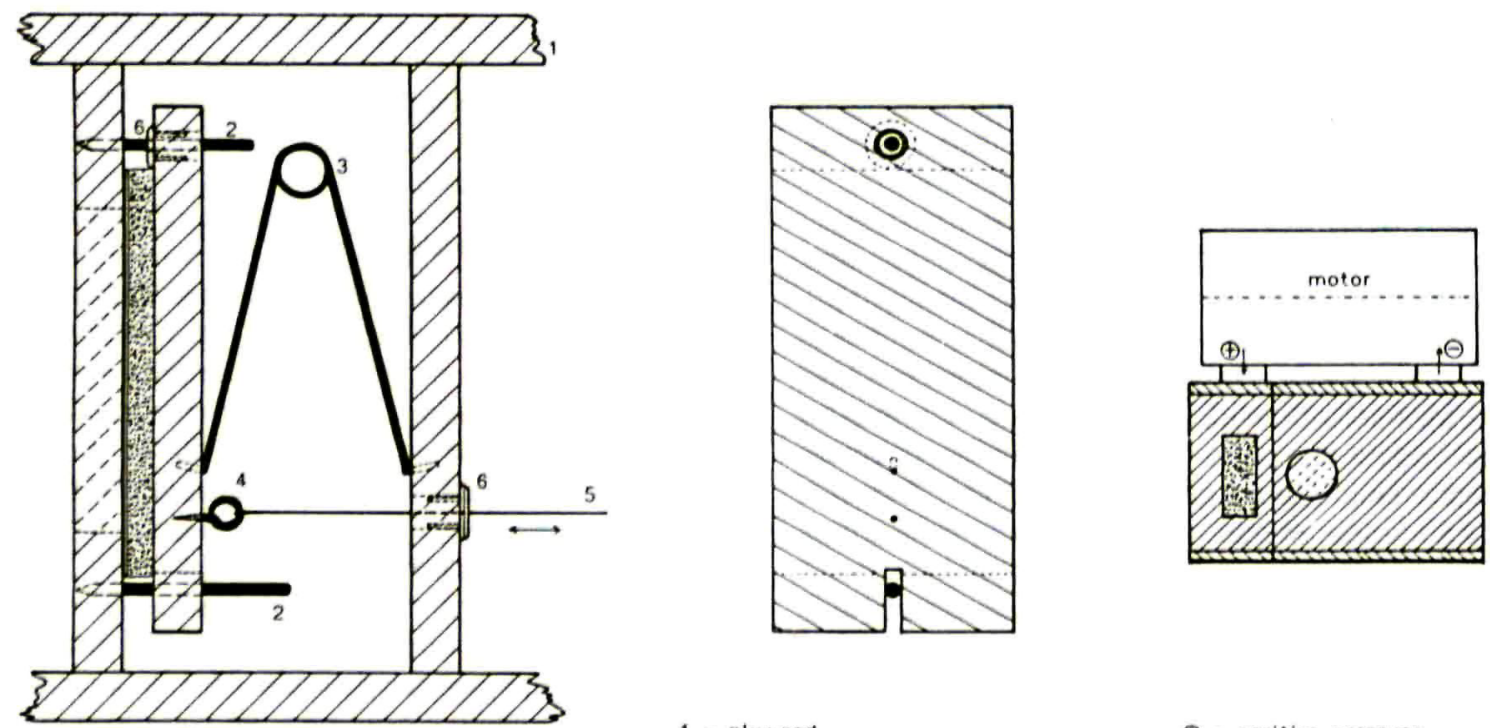

1 - plywood

2 = pins made of tinned brass

3 = compass spring

4 - small screw eye

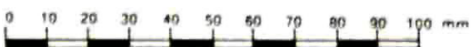

5 = nylon cord

6 - small plastic bungs

( ) = positive pressure

$\Theta=$ negative pressure

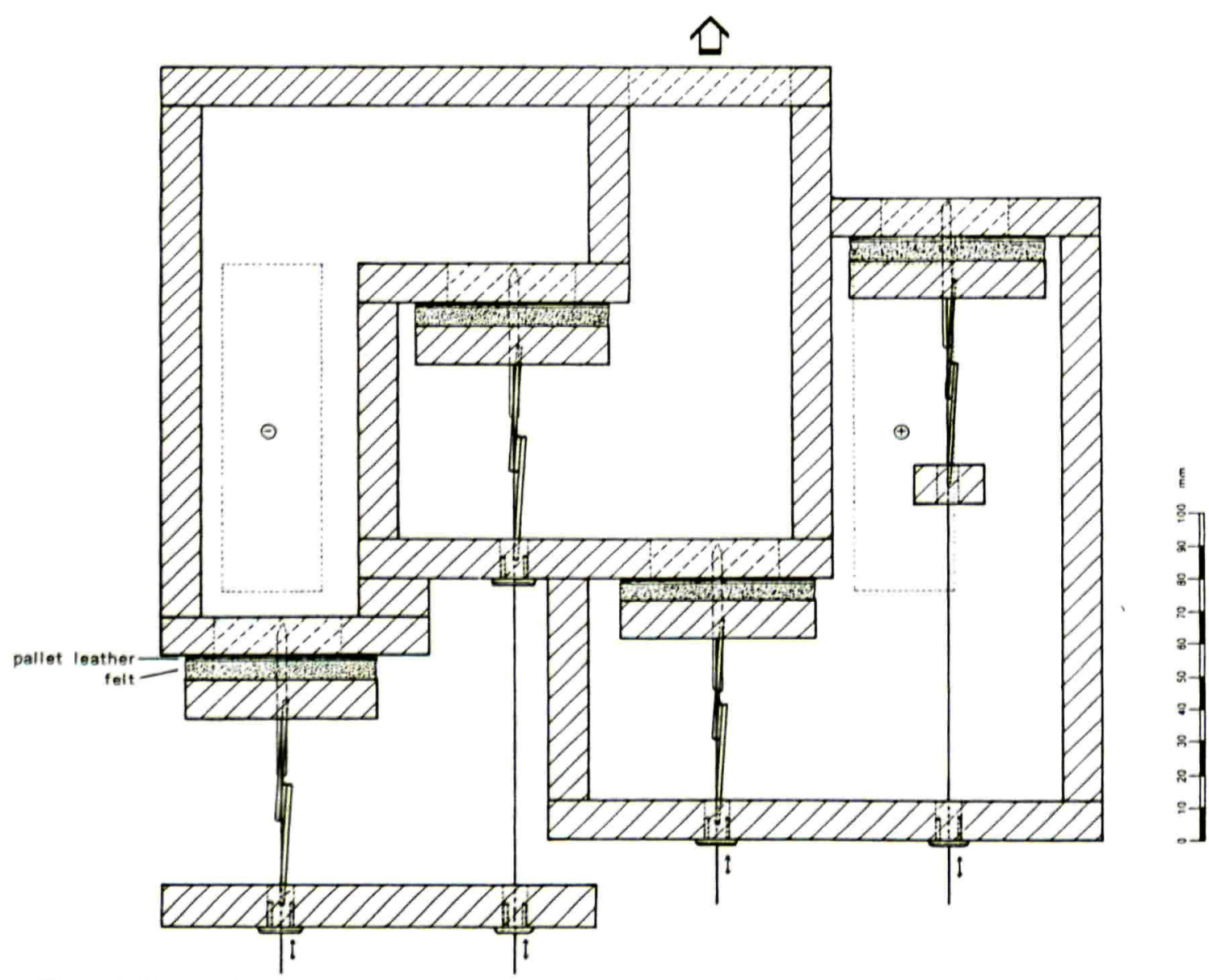

Figure 3. Sketch of the valve construction (upper left and upper middle part), the valve configuration (lower part), and the valve-motor complex (upper right part). 
The opening and closing of the valves are triggered by the up-stroke of the R-wave of the electrocardiogram. Each time an R-wave is detected, a Beckman 9806 cardiotachometer coupler generates a square wave pulse (Q-output). This square wave is fed into the computer (via a TTL circuit attached to the digital input), which then determines the time within $1 \mathrm{~ms}$ it takes for the R-wave to appear. For stimulatory trials (systolic suction/diastolic excess pressure) the cuff pressure begins to drop $100 \mathrm{~ms}$ after the occurrence of the R-wave. About $2 / 3$ of the final negative pressure is achieved $180 \mathrm{~ms}$ later. This constellation of valves, and thus the negative pressure, remains for an interval lasting exactly as long as half of the previous trial's mean interbeat interval (IBI) minus $100 \mathrm{~ms}$ (IBI/2-100 ms). After this time interval has elapsed, a positive cuff pressure is applied for exactly the same duration. Then the valves are switched to the neutral state (Valves 3 and 4 open, 1 and 2 closed) until the next $R$-wave is detected. Then the whole sequence is started again, resulting in a continuous wave of bipolar pressure pulses. In the present experiment, this continuous wave was presented for periods of $6 \mathrm{~s}$ each. Within a 6-s period, 4-9 (depending on the heart rate) sequences of positive and negative cuff pressures were presented.

For control trials the sequence of negative and positive pressure pulses is reversed in time: each $\mathrm{R}$-wave triggers a positive cuff pressure (again for $50 \%$ of the duration of the previous trial's mean minus $100 \mathrm{~ms}$ ). This period is followed by a pressure period pulse of equal duration. If the next $R$ wave occurs prior to the completion of the bipolar pressure pulse, $100 \mathrm{~ms}$ later the current pulse is interrupted and the next one is initiated.

To prevent subjects from differentiating the two conditions, each trial begins with a negative pressure burst. If an R-wave occurs before the sequence of pressure changes has been completed, the sequence is initiated anew.

\section{EXPERIMENT 1}

The aim of Experiment 1 was to test the influence of both PRES conditions on heart rate in subjects whose R-waves triggered the cuff pressure changes. It was hypothesized that trials including systolic negative cuff pressure and diastolic positive cuff pressure should activate the carotid sinus baroreceptors more than trials consisting of diastolic negative and systolic positive cuff pressure. This differential activation should be followed by differential heart rate responses: the first condition was predicted to lower heart rate more substantially than the second condition.

\section{Methods}

\section{Subjects}

Eleven male staff members (aged 27-48 years) from our department served as subjects in the present experiment. All subjects were free of cardiovascular abnormalities and none were currently on medication.

\section{Physiological Recordings and Procedure}

The ECG was recorded from electrodes attached to both the left lower rib cage and the right clavicle and grounded on the right clavicle. The raw ECG signal was fed into a cardiotachometer module (Beckman) for R-wave detection. Heart rate was calculated from the interbeat interval and stored on hard disc.

A computer program, written using the ASYST language on an MS/DOS computer, initiated 64 trials, each trial consisting of a 1-s baseline period and a 6s stimulation period. Thirty-two stimulatory trials with systolic suction/diastolic excess pressure ("stimulation") and 32 control trials with systolic excess pressure/diastolic suction ("control") were presented in a pseudorandomized sequence with an intertrial interval varying from $10-16 \mathrm{~s}$ in length. The computer was programmed for data collection (heart rate) and the timing of the pressure changes.

For each subject heart rate responses were averaged separately for the stimulation and control trials. The means during the second half of each trial (seconds $4,5,6)$ were submitted to an ANOVA in which Condition (stimulation vs. control) served as a within-subjects factor.

\section{Results}

In all 11 subjects, the stimulation trials evoked greater heart rate decelerations than the control trials. The upper part of Figure 4 depicts the grand mean of the heart rate for both conditions. The mean of the second half of the active interval (seconds 4-6 after beginning the pressure changes) was significantly different between conditions $(F(10)=$ $9.41, p=.01)$. During the second half of stimulation trials, mean heart rate decreased by $4.3 \mathrm{bpm}(\mathrm{SD}=$ 4.3), referred to a 1-s prestimulation baseline. During the control condition, on the other hand, heart rate was not significantly influenced and showed a change of $.3 \mathrm{bpm}(\mathrm{SD}=.6)$, referred to baseline. To test a relationship between baseline heart rate level and stimulation induced heart rate changes, a correlation between heart rate responses to both stimulation conditions and baseline heart rate (mean heart rate during the last second before the beginning of the stimulation) was computed. The correlation was not significant $(r=.43, t(10)=1.41)$. This can also be seen in Figure 5. 

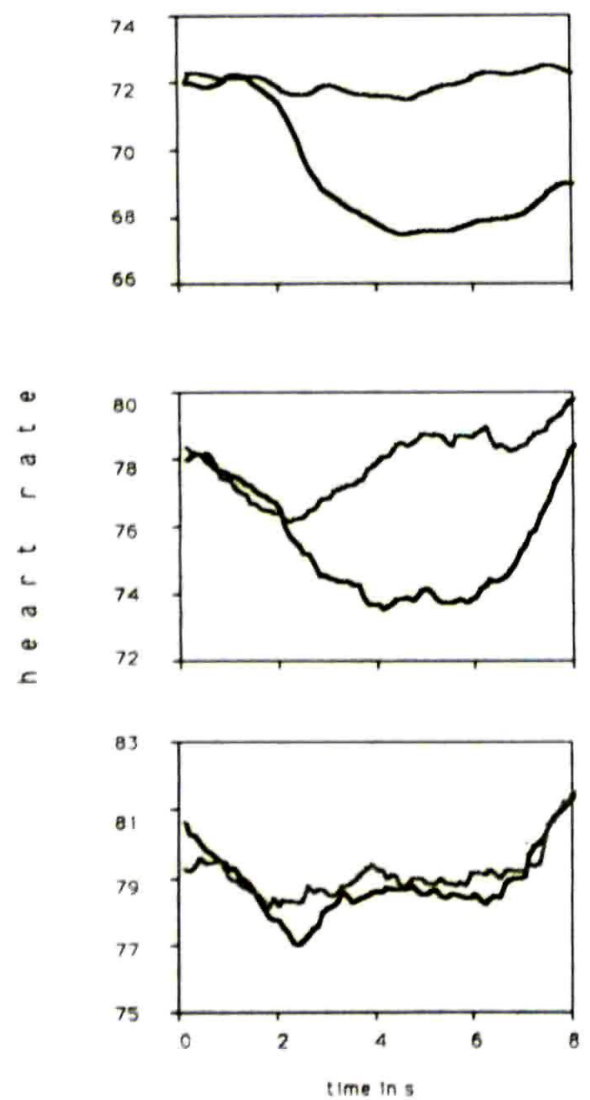

Figure 4. Grand mean of heart rate responses to stimulation (solid line) and control (dashed line) trials. Baroreceptor stimulation starts at Second 1. Top. Data from Experiment 1 including 11 subjects. Middle. Data from the experimental group of Experiment 2 including 15 subjects. Bottom. Data from the yoked control group of Experiment 2 including 15 subjects.

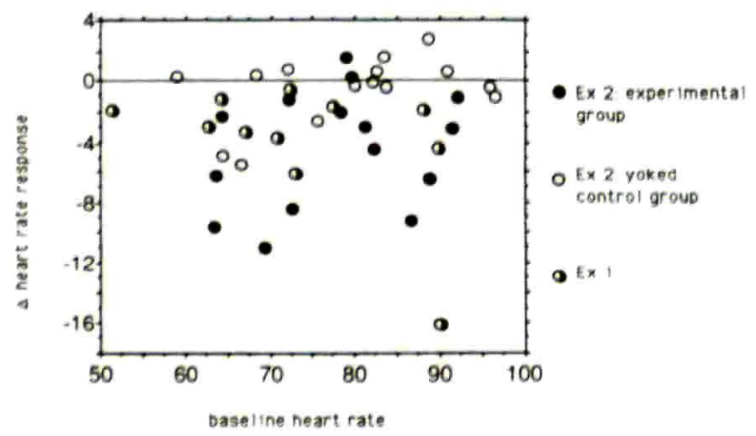

Figure 5. Baseline heart rate and differential heart rate responses for each individual subject in bpm.

\section{Discussion}

Activation of the baroreceptors elicits the baroreceptor reflex, resulting in heart rate decelerations. Presently, decelerations were observed when systolic suction pulses were followed by diastolic excess pressure changes, but not when the reversed sequence was presented. This result confirmed the hypothesis. Surprisingly, there were great interindividual differences in the magnitude of the heart rate responses to PRES. A dependence of the magnitude of the heart rate response upon absolute heart rate could be suspected because the absolute heart rate level determines how long one suction period is and how many of these periods within one trial will appear. For this reason a correlational analysis between heart rate response and heart rate level was computed. This correlation was not significant, and as can also be inferred from Figure 5, heart rate level seems to have little influence on the heart rate responses elicited by PRES.

Whether the observed heart rate differentiation between the two PRES conditions was a consequence of the cuff pressure sequence itself or whether it originated from the relationship between this pressure sequence and the heart cycle, as postulated, could not be determined from Experiment 1. Experiment 2 was designed to resolve this question. The effects of both PRES conditions on heart rate in a group of experimental subjects whose heart cycle actually triggered PRES were compared with these effects in a group of yoked control subjects who received PRES stimulation independently of their cardiac cycle.

\section{EXPERIMENT 2}

To examine the influence of the time relationship between the cardiac cycle and cuff pressure changes, a second experiment was conducted. In this experiment two subjects were always tested simultaneously: The experimental subject triggered with her/his ECG the cuff pressure changes of both subjects. The stimulation method was the same as described above. The yoked control subjects received the same cuff pressure changes as the experimental subjects, but these sequences were not related to their own heart cycles. It was hypothesized that the two PRES conditions would elicit different heart rate responses in the experimental subjects alone, and not in the yoked control subjects.

\section{Methods \\ Subjects}

Thirty students of Psychology, aged 19-25 years, served as subjects in the present experiment. They received course credit for their participation. All subjects were free of any cardiovascular diseases and any medication intake. The paired subjects were placed in the same room. They were not informed about the assignment to two different groups. The between-subjects factor Group (experimental vs. yoked control) was added to the ANOVA. 


\section{Physiological Recordings and Procedure}

Two subjects were investigated at a time. Both were attached with a neck cuff, connected to two identical but separate pressure machines as described above. The ECG was recorded in both subjects the same way as described for Experiment 1. However, the ECG of only one subject (the experimental subject) triggered both machines. The duration of the stimulation period was shortened to $4.5 \mathrm{~s}$.

\section{Results}

A significant Group $\times$ Condition interaction for heart rate indicated that the two stimulation conditions had quite different effects for the two groups: $F(1,28)=16.32, p<0.001$. As predicted, heart rate responses differed between the two stimulation conditions only in the experimental group $(F(1,14)=$ $31.00 ; p<0.001$ ), not in the yoked control group $(F(1,14)=0.32)$. The middle and bottom panels of Figure 4 depict the heart rate responses across all subjects, separated for conditions and groups. A correlational analysis between the individual heart rate baselines and heart rate responses to the two PRES conditions was not significant, either in the experimental or in the yoked control group. Figure 5 shows the relationships between baseline heart rate and the difference in heart rate responses between the two PRES conditions.

\section{Discussion}

The results of Experiment 2 support the hypothesis that heart rate response differences between the two PRES conditions are dependent upon the time relationship of the cuff pressure changes to the heart cycle: Subjects receiving the same cuff pressure changes but unrelated to their own heart cycle show no differentiation in heart rate response to the two PRES conditions. This result further validates PRES as a baroreceptor stimulation method including a control condition. Nevertheless, as an inspection of Figure 5 shows, two subjects in the yoked control group showed a marked heart rate differentiation of more than $4 \mathrm{bpm}$. It seems that these two subjects had heart rate levels similar to those of their experimental counterparts. In case of a similar heart rate level it is more likely that the heart rates become synchronized through the stimulation. In this case the PRES conditions may work even in the yoked control subjects. Inspection of
Figure 5 also shows that there was one subject in the experimental group with a heart rate differentiation in the wrong direction. This demonstrates that the PRES technique seems not to work in all subjects adequately.

\section{GENERAL DISCUSSION}

Results of Experiment 1 demonstrate a clear differentiation in heart rate response to the two PRES conditions. The great interindividual differences in heart rate responses could not be explained with the absolute heart rate level. Other uncontrolled factors may account for this phenomenon, however. For example, the different thickness of the fatty tissue of the neck could influence the amount of pressure transduction to the carotid sinus and thus influence the efficacy of the stimulation method. Additionally, there exists different baroreceptor sensitivity in different people, dependent on tonic blood pressure and age. Neither variable has been controlled in the present studies.

Results of Experiment 2 replicated the differentiation in heart rate response to the two PRES conditions for the experimental subjects. If both PRES conditions are presented independently from the heart cycle, a heart rate deceleration of about 2 bpm can be observed, with no differentiation between PRES conditions. This result demonstrates that the effects of PRES depend upon the timing of the stimulation within the cardiac cycle. Although direct measures of baroreceptor activity in humans cannot be performed noninvasively, the results are consistent with the notion advanced here that the baroreceptors respond differentially to cardiac cycle dependent PRES conditions, and that the baroreceptor cardiac cycle effects mediate the observed heart rate responses.

In sum, we hypothesized that baroreceptors are more sensitive to suction bursts during systole than during diastole. Greater heart rate decelerations were observed during trials with systolic suction, as hypothesized. A more specific measure of baroreceptor activation would be worthwhile to evaluate further the possibilities and restrictions of the PRES method. In the meantime, we recommend PRES as a baroreceptor stimulation technique for psychophysiological investigations, because it seems to be the only noninvasive method currently available that allows for psychological control conditions.

\section{REFERENCES}

Dworkin, B. (1988). Hypertension as a learned response: The baroreceptor reinforcement hypothesis. In T. Elbert, W. Langosch, A. Steptoe, \& D. Vaitl (Eds.), Behavioral medicine in cardiovascular disorders (pp. 1747). Chichester: John Wiley \& Sons.
Dworkin, B., Filewich, R.J., Miller, N.E., Craigmyle, N., \& Pickering, T.G. (1979). Baroreceptor activation reduces reactivity to noxious stimulation: Implications for hypertension. Science, 205, 1299-1301.

Eckberg, D., Cavanaugh, M., Mark, A., \& Abboud, F. 
(1975). A simplified neck suction device for activation of carotid baroreceptors. Journal of Laboratory \& Clinical Medicine, 85, 167-173.

Eckberg, D.L. (1976). Temporal response patterns of the human sinus node to brief carotid baroreceptor stimuli. Journal of Physiology. 258, 769-782.

Elbert, T., Rockstroh, B., Lutzenberger, W., Kessler, M., Pietrowsky, R., \& Birbaumer, N. (1988). Baroreceptor stimulation alters pain sensation depending on tonic blood pressure. Psychophysiology, 25, 25-29.

Kirchheim, H. (1976). Systemic arterial baroreceptor reflexes. Physiological Reviews, 56, 100-176.

Lacey, J., \& Lacey, B. (1970). Some autonomic-central nervous system interrelationships. In P. Black (Ed.), Physiological correlates of emotion (pp. 205-227). New York: Academic Press.

Larbig, W., Elbert, T., Rockstroh, B., Lutzenberger, W., \& Birbaumer, N. (1985). Elevated blood pressure and reduction of pain sensitivity. In J.F. Orlebeke, G. Mulder, \& L.P.J. van Doornen (Eds.), The psychophysiology of cardiovascular control (pp. 113-122). New York: Plenum.
Mancia, G., Ferrari, A., Gregorini, L., Valentini, R., Ludbrook, J., \& Zanchetti, A. (1977). Circulatory reflexes from carotid and extracarotid baroreceptor areas in man. Circulation Research, 41, 309-315.

Randich, A., \& Maixner, W. (1984). Interactions between cardiovascular and pain regulatory systems. Neuroscience \& Biobehavioral Reviews, 8, 343-367.

Rau, H., Elbert. T, Lutzenberger, W., Eves, F., Rockstroh, B., Larbig, W., \& Birbaumer, N. (1988). Pavlovian conditioning of peripheral and central components of the baroreceptor reflex. Journal of Psychophysiology. 2, 119-127.

Schweizer, R.M., Roth, W.T., \& Elbert, T. (1991). Effect of two $\beta$-blockers on stress during mental arithmetic. Psychopharmacology, 105, 575-577.

Toon, P.D., Bergel, D.H., \& Johnston, D.W. (1984). The effect of modification of baroreceptor activity on reaction time. Psychophysiology, 21, 486-494.

Vaitl, D., \& Gruppe, H. (1990). Changes in hemodynamics modulate electrical brain activity. Journal of Psychophysiology, 4, 41-49. 